\title{
Time-resolved photometry of the nova remnants DM Gem, CP Lac, GI Mon, V400 Per, CT Ser and XX Tau^
}

\author{
P. Rodríguez-Gil ${ }^{1,2}$ and M. A. P. Torres ${ }^{3,4}$ \\ 1 Department of Physics, University of Warwick, Coventry CV4 7AL, UK \\ e-mail: Pablo. Rodriguez-Gil@warwick .ac.uk \\ 2 Instituto de Astrofísica de Canarias, Vía Láctea, s/n, La Laguna, 38205 Santa Cruz de Tenerife, Spain \\ ${ }^{3}$ Harvard-Smithsonian Center for Astrophysics, 60 Garden St, Cambridge, MA 02138, USA \\ ${ }^{4}$ Physics Department, University College, Cork, Ireland
}

Received 16 April 2004 / Accepted 7 October 2004

\begin{abstract}
We present the first results of a photometric survey of poorly studied nova remnants in the Northern Hemisphere. The main results are as follows: DM Gem shows a modulation at $0.123 \mathrm{~d}$ (probably linked to the orbit) and rapid variations at $\sim 22 \mathrm{~min}$. A moderate resolution spectrum taken at the time of the photometric observations shows intense He II $\lambda 4686$ and Bowen emission, characteristic of an intermediate polar or a SW Sex star. Variability at $0.127 \mathrm{~d}$ and intense flickering (or quasi-periodic oscillations) are the main features of the light curve of CP Lac. A 0.1-mag dip lasting for 45 min is observed in GI Mon, which could be an eclipse. A clear modulation (probably related to the orbital motion) either at $0.179 \mathrm{~d}$ or $0.152 \mathrm{~d}$ is observed in the $B$-band light curve of V400 Per. The results for CT Ser point to an orbital period close to $0.16 \mathrm{~d}$. Intense flickering is also characteristic of this old nova. Finally, XX Tau shows a possible periodic signal near $0.14 \mathrm{~d}$ and displays fast variability at $\sim 24 \mathrm{~min}$. Its brightness seems to be modulated at $\sim 5 \mathrm{~d}$. We relate this long periodicity to the motion of an eccentric/tilted accretion disc in the binary.
\end{abstract}

Key words. accretion, accretion discs - stars: binaries: close - novae, cataclysmic variables

\section{Introduction}

Cataclysmic variable stars $(\mathrm{CVs})$ exhibit their most extreme outbursting behaviour in classical novae. These explosive events are the consequence of thermonuclear runaways in the hydrogen envelope of the white dwarf as a result of continuous mass accretion on to its surface (Starrfield et al. 1976). It takes decades for a nova to return to its quiescent (prenova/postnova) state, where most of them behave like nova-like CVs. Comprehensive reviews on classical novae in particular and CVs in general can be found in Bode \& Evans (1989) and Warner (1995), respectively.

So far there is a large number of nova remnants that have received little or no attention at all due to their faintness. Because of the lack of study, a crucial parameter such as the orbital period is currently undetermined for the majority of these quiescent novae. Many of these systems might show the hallmarks

* Based in part on observations made with the Jacobus Kapteyn Telescope, which was operated on the island of La Palma by the Isaac Newton Group in the Spanish Observatorio del Roque de los Muchachos of the Instituto de Astrofísica de Canarias (IAC), and on observations made with the IAC80 telescope, operated on the island of Tenerife by the IAC in the Spanish Observatorio del Teide of the IAC. Observations were also obtained at the FLWO Observatory, a facility of the Smithsonian Institution. of the orbital motion in their optical light curves in the form of pure orbital modulations, superhumps, ellipsoidal modulations, eclipses, etc. Thus, time-resolved photometric monitoring provides an easy way to obtain a reliable estimate of their orbital periods.

The main goals of this long-term project are to obtain accurate estimates of the orbital periods of poorly studied novae remnants as the necessary input for efficient time-resolved spectroscopic monitoring, and to search for photometric behaviour characteristic of the different $\mathrm{CV}$ sub-classes. In this paper we present the first results for six novae remnants.

\section{Observations and data reduction}

Four telescopes were used to obtain the data presented in this paper: the 1.0-m Jacobus Kapteyn Telescope (JKT) on La Palma, the 0.82-m IAC80 telescope on Tenerife, and the 1.2-m and 1.5-m telescopes at the Fred Lawrence Whipple Observatory (FLWO) in Arizona. A log of observations is presented in Table 1, where the novae are listed alphabetically by constellation name. Details on the instrumental setups employed during the observations are given in what follows:

1. The photometric data obtained with the $J K T$ were extracted from CCD images collected without filter (white light) 
Table 1. Log of observations.

\begin{tabular}{|c|c|c|c|}
\hline UT date & $\begin{array}{c}\text { Coverage } \\
\text { (h) }\end{array}$ & Filter/Grating & $\begin{array}{l}\text { Exp. } \\
\text { (s) }\end{array}$ \\
\hline \multicolumn{4}{|c|}{ DM Gem } \\
\hline \multicolumn{4}{|c|}{ 1.2-m FLWO CCD photometry } \\
\hline 2004 Mar. 11 & 3.98 & White light & 30 \\
\hline 2004 Mar. 14 & 1.28 & White light & 30 \\
\hline 2004 Mar. 15 & 4.87 & White light & 30 \\
\hline 2004 Mar. 16 & 4.87 & White light & 30 \\
\hline \multicolumn{4}{|c|}{ 1.5-m FLWO, FAST spectroscopy } \\
\hline 2004 Mar. 15 & - & $300 \mathrm{l} / \mathrm{mm}$ & 1200 \\
\hline \multicolumn{4}{|c|}{ CP Lac } \\
\hline \multicolumn{4}{|c|}{ 1.0-m JKT CCD photometry } \\
\hline 2002 Oct. 28 & 4.80 & White light & 10 \\
\hline 2002 Oct. 29 & 6.72 & White light & 10 \\
\hline 2002 Oct. 30 & 6.85 & White light & 10 \\
\hline 2002 Oct. 31 & 5.73 & White light & 10 \\
\hline \multicolumn{4}{|c|}{ GI Mon } \\
\hline \multicolumn{4}{|c|}{ 1.0-m JKT CCD photometry } \\
\hline 2002 Nov. 02 & 3.62 & White light & 15 \\
\hline 2002 Nov. 04 & 3.88 & White light & 15 \\
\hline \multicolumn{4}{|c|}{ 1.2-m FLWO CCD photometry } \\
\hline 2004 Mar. 17 & 3.84 & White light & 20 \\
\hline 2004 Mar. 18 & 4.04 & White light & 20 \\
\hline \multicolumn{4}{|c|}{ V400 Per } \\
\hline \multicolumn{4}{|c|}{ 1.0-m JKT CCD photometry } \\
\hline 2002 Nov. 03 & 1.57 & $B$ & 360 \\
\hline 2002 Nov. 04 & 6.03 & $B$ & 360 \\
\hline \multicolumn{4}{|c|}{ 0.82-m IAC80 CCD photometry } \\
\hline 2003 Jan. 03 & 4.94 & $B$ & 1200 \\
\hline \multicolumn{4}{|c|}{ CT Ser } \\
\hline \multicolumn{4}{|c|}{ 1.2-m FLWO CCD photometry } \\
\hline 2004 Mar. 09 & 3.18 & White light & 20 \\
\hline 2004 Mar. 10 & 2.17 & White light & 20 \\
\hline 2004 Mar. 14 & 3.02 & White light & 20 \\
\hline 2004 Mar. 15 & 3.70 & White light & 20 \\
\hline 2004 Mar. 16 & 4.50 & White light & 20 \\
\hline \multicolumn{4}{|c|}{ XX Tau } \\
\hline \multicolumn{4}{|c|}{ 1.0-m JKT CCD photometry } \\
\hline 2002 Oct. 28 & 4.90 & White light & 240 \\
\hline 2002 Oct. 29 & 4.20 & White light & 150 \\
\hline 2002 Oct. 30 & 4.08 & White light & 180 \\
\hline 2002 Oct. 31 & 3.75 & White light & 150 \\
\hline 2002 Nov. 02 & 6.77 & White light & 150 \\
\hline
\end{tabular}

using the $2048 \times 2048$ pixel $^{2}$ SITe CCD camera, with the exception of V400 Per for which a Johnson $B$ filter was in place.

2. Additional photometry of V400 Per was obtained with the IAC80 telescope. The telescope was equipped with the $1024 \times 1024$ pixel $^{2}$ Thomson CCD camera and a Johnson $B$ filter.

3. The 1.2-m telescope at FLWO was equipped with the 4-Shooter mosaic CCD camera which consists of an array of four $2048 \times 2048$ pixel $^{2}$ chips. Only the CCD \#3 was used to image the targets. All the images were obtained without filter.

4. A 20-min optical spectrum of DM Gem was obtained using the FAST spectrograph (Fabricant et al. 1998) attached to the 1.5-m telescope at the FLWO. Use of a $3^{\prime \prime}$ slit and the 300 line $\mathrm{mm}^{-1}$ grating provided a wavelength coverage of $\lambda \lambda 3800-7500$ with a resolution of $\sim 7 \AA(F W H M)$.

In order to improve the time resolution of our photometry, the detectors were operated in $2 \times 2$ binning mode and only a small window containing the target and a suitable number of comparison stars was read out. All the reduction processes and photometry extraction were done with IRAF $^{1}$. After correcting the raw images for the effects of bias level and flat field structure, the instrumental magnitudes of each target and three comparison stars were computed by means of aperture photometry. An aperture radius of 1.5 times the full width at half maximum ( $F W H M)$ of the typical seeing profile was used (Naylor 1998). The sky background level was measured inside an 8-pixel width annulus with internal radius safely away from the target aperture. The scatter in the comparison light curves shows that the differential photometry is accurate to $<5$ per cent in all cases.

The spectrum of DM Gem was extracted and wavelength calibrated using standard IRAF tasks as part of the CfA spectroscopic data pipeline (Kurtz \& Mink 1998). Flux standards taken on the same night were used to correct for the instrumental response, but the night was not photometric for an absolute flux calibration.

\section{Results}

\subsection{DM Gem}

DM Gem (Nova Geminorum 1903) was discovered at $m_{\mathrm{pg}} \sim 8.0$ on 1903 March 16 by Professor Turner. Examination of archival photographic plates showed the nova at $m_{\mathrm{pg}} \sim 5$ on 1903 March 6 (Pickering et al. 1903). A $t_{2}=6$ days (Schmidt 1957) is consistent with a very fast light curve, where $t_{2}\left(\right.$ or $\left.t_{3}\right)$ is the time in days that the light curve takes to decline 2 (or 3 ) magnitudes below maximum brightness. A magnitude of $V=17.38$ for the postnova was reported by Szkody (1994).

We observed DM Gem on the nights of 2004 March 11, 14, 15 and 16 at the FLWO. The light curves (Fig. 1) seem to show a non-sinusoidal variation at about $3 \mathrm{~h}$ (see e.g. the 2004 March 16 light curve). We have combined all the data to compute the Scargle periodogram (Scargle 1982) presented in the upper panel of Fig. 2. The strongest peak near $3 \mathrm{~h}$ is centred at $0.123 \pm 0.002 \mathrm{~d}$. Hereafter the quoted errors represent half the FWHM of the corresponding peak as measured by using a Gaussian fit. The peaks at lower frequencies are centred at multiples of $1 \mathrm{~d}^{-1}$ as a consequence of the observing window. The peak at $0.123 \mathrm{~d}$ is close to this region of false frequencies. However, the fact that we see a modulation at this period in

\footnotetext{
1 IRAF is distributed by the National Optical Astronomy Observatories, which is operated by the Association of Universities for Research in Astronomy, Inc., under contract with the National Science Foundation.
} 

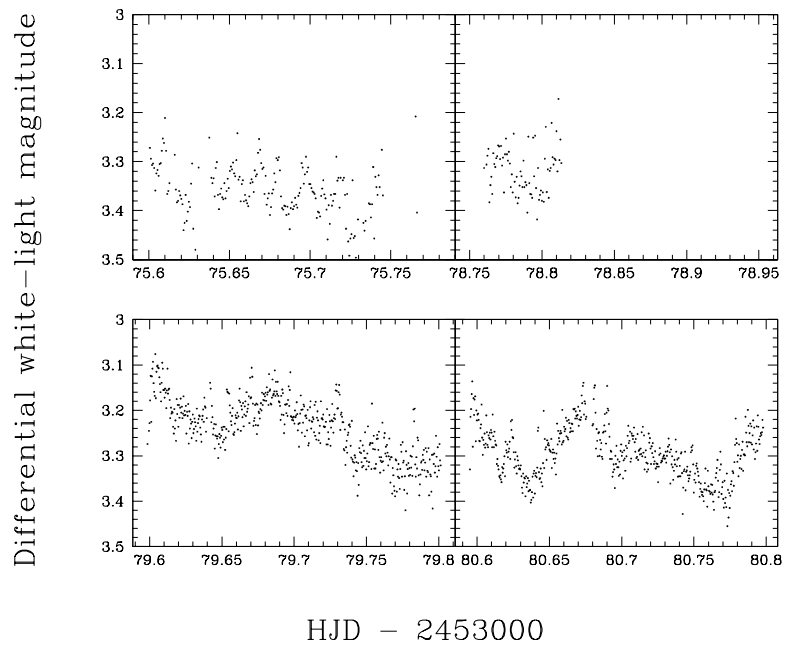

Fig. 1. Individual light curves of DM Gem obtained at the FLWO. From left to right and from top to bottom: 2004 March 11, 14, 15 and 16.
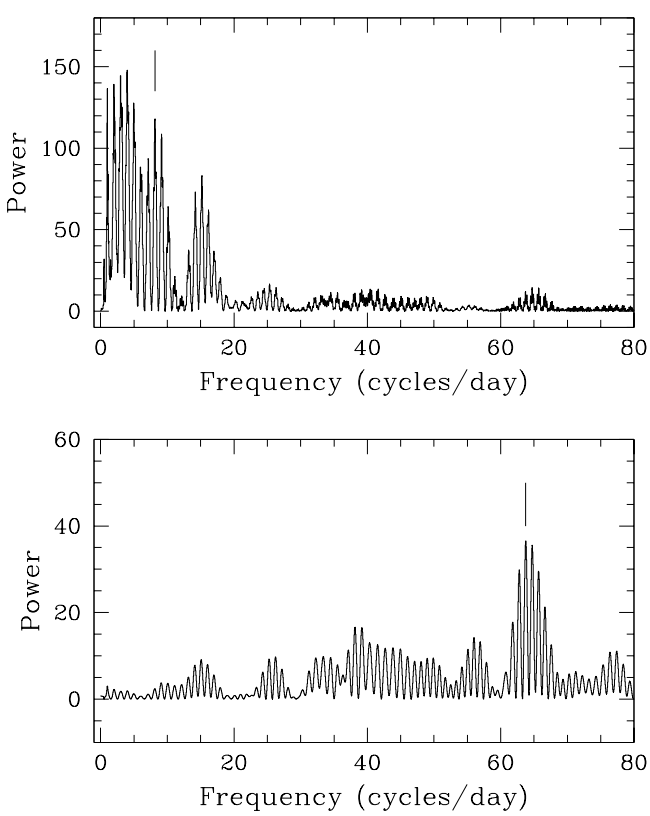

Fig. 2. Top: Scargle periodogram for DM Gem. Bottom: Scargle periodogram after removing the 0.123 -d signal.

two of the light curves indicates that it might be real. Note that Lipkin et al. (2000) found the same signal, which was interpreted as a quasi-periodic oscillation.

The light curve of DM Gem also displays short-term variations at a time scale of $\sim 20 \mathrm{~min}$ (see e.g. the 2004 March 11 light curve). In order to search for this periodicity, a new Scargle periodogram was computed after removing the 0.123 -d signal from the individual light curves by subtracting a smoothed version of each light curve (bottom panel of Fig. 2). The strongest peak is centred at $22.58 \pm 0.09 \mathrm{~min}$.

A single spectrum of DM Gem was obtained on the night of 2004 March 15. The spectrum (Fig. 3) shows single-peaked emission lines of He II $\lambda 4686$ (equivalent width, $E W=5 \pm$ $\left.1 \AA, F W H M=1088 \pm 130 \mathrm{~km} \mathrm{~s}^{-1}\right)$, the Bowen blend at $\lambda \lambda 4640-4650\left(E W=3 \pm 1 \AA, F W H M=1100 \pm 260 \mathrm{~km} \mathrm{~s}^{-1}\right)$

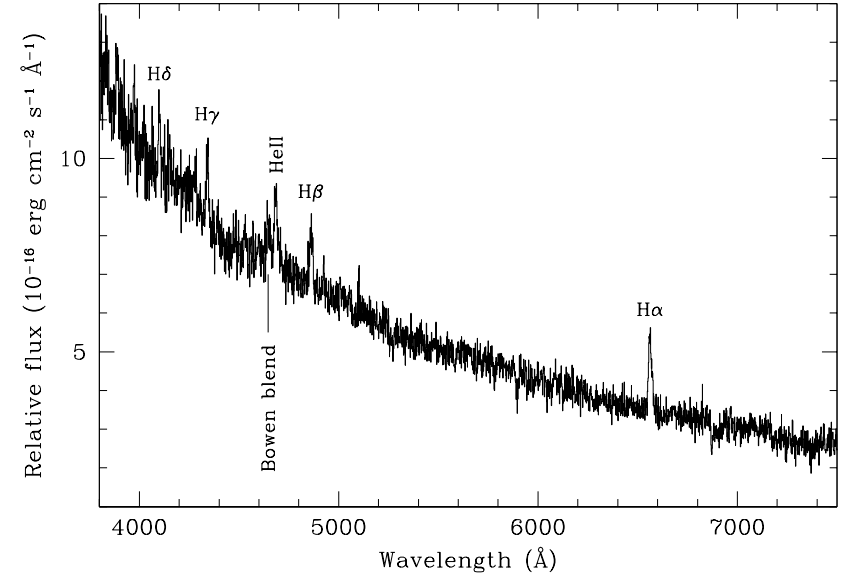

Fig. 3. Optical spectrum of DM Gem.

and the Balmer series up to $\mathrm{H} \delta: \mathrm{H} \alpha(E W=11 \pm 1 \AA, F W H M=$ $\left.914 \pm 50 \mathrm{~km} \mathrm{~s}^{-1}\right), \mathrm{H} \beta(E W=4 \pm 1 \AA, F W H M=1110 \pm$ $\left.120 \mathrm{~km} \mathrm{~s}^{-1}\right), \mathrm{H} \gamma\left(E W=3 \pm 1 \AA, F W H M=900 \pm 140 \mathrm{~km} \mathrm{~s}^{-1}\right)$ and $\mathrm{H} \delta\left(E W=2 \pm 1 \AA, F W H M=800 \pm 140 \mathrm{~km} \mathrm{~s}^{-1}\right)$. The unresolved $\mathrm{NaD}$ doublet at $\lambda 5890, \lambda 5896$ is also evident with an $E W$ of $-1.5 \pm 1 \AA$. This large $E W$ prevents us from using the Munari \& Zwitter (1997) calibration to derive the reddening from this interstellar feature as the $\mathrm{NaD} E W /$ reddening relationship does not change appreciably for $E W \leq-0.5 \AA$. A powerlaw fit to the observed spectrum in the range $\lambda \lambda 4000-6900$ (after masking the emission lines) provides a power-law index of $-2.19 \pm 0.03$.

The most remarkable spectral feature in DM Gem is the strong He II $\lambda 4686$ emission line (He II/H $\beta \gtrsim 1)$, which indicates the presence of a source of ionising photons within the binary. This, together with the (unconfirmed) rapid photometric variability, are characteristic signatures of the Intermediate Polar (IP) CVs and many SW Sex stars. The latter have been proposed as magnetic accretors (Rodríguez-Gil et al. 2001a; Rodríguez-Gil 2003). Intense He II $\lambda 4686$ emission has been observed e.g. in the IP systems V1223 Sgr (Steiner et al. 1981) and AO Psc (Cordova et al. 1983), and in the SW Sex stars BT Mon (Williams 1983) and V348 Pup (Rodríguez-Gil et al. 2001b).

\subsection{CP Lac}

CP Lac (Nova Lacertae 1936) was discovered visually by Kazuaki Gomi during the 1936 June 18 total solar eclipse. The nova reached maximum light $(V \simeq 2.0$; Bertaud 1945) two days later, being $\sim 13.5 \mathrm{mag}$ brighter than the pre/postnova (Robinson 1975). The light curve during the decline phase after the nova explosion showed that CP Lac was a very fast nova with $t_{2}=5.3$ days, $t_{3}=9.8$ days (Bertaud 1945).

The postnova optical spectrum (Ringwald et al. 1996) shows Balmer and He I emission lines. Also present are the He II $\lambda 4686$ emission line and the Bowen blend, both with similar strength. A multi-year (1991-1997) light curve (Honeycutt et al. 1998) shows six stunted outbursts characterised by having smaller amplitude than typical dwarf novae outbursts. 

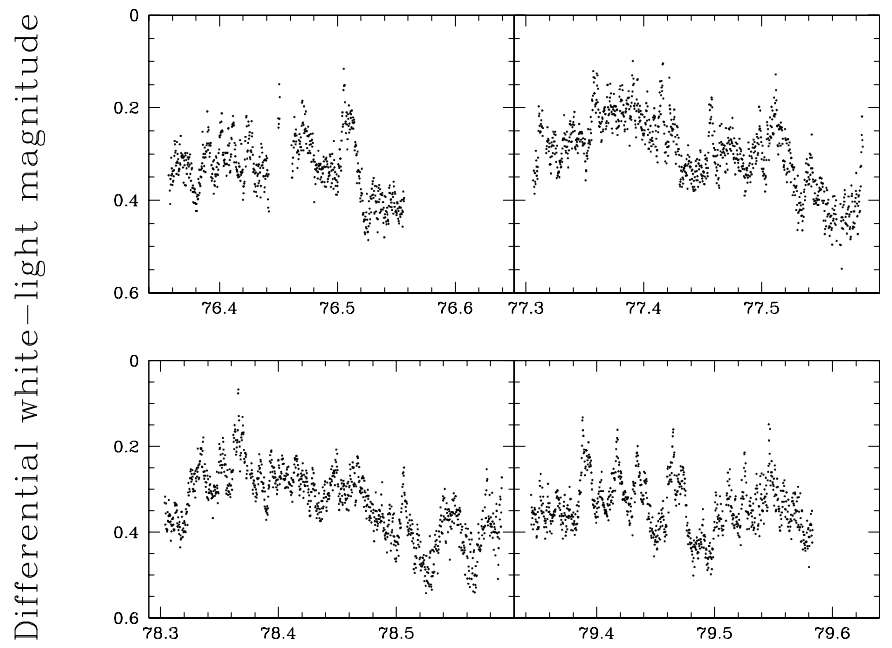

HJD - 2452500

Fig. 4. Unfiltered $J K T$ light curves of CP Lac. Top panel: 2002 October 28 (left) and 29 (right). Bottom panel: 2002 October 30 (left) and 31 (right).

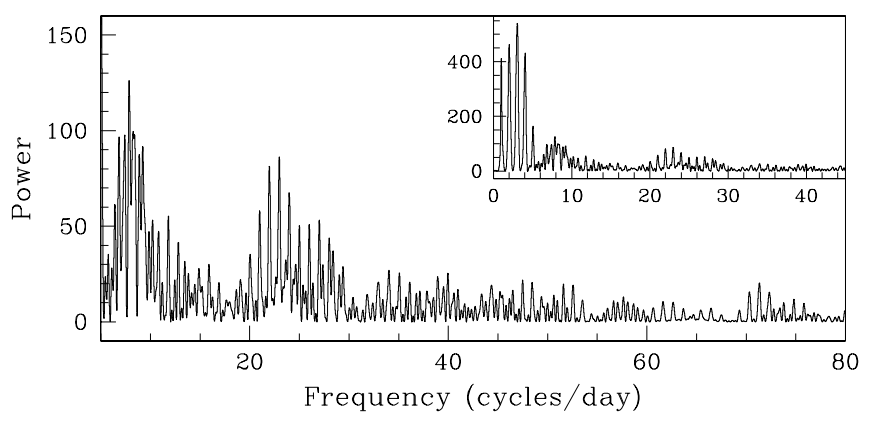

Fig. 5. Scargle periodogram computed from all the CP Lac light curves (nightly averages subtracted). The strong peaks in the low frequency regime of the periodogram $\left(v<5 \mathrm{~d}^{-1}\right)$ are very likely due to the observing window.

In Fig. 4 we present the unfiltered light curves of CP Lac obtained on 2002 October 28-31 with the JKT on La Palma. The light curves are dominated by intense and rapid variations (tens of minutes time scale) with an amplitude of $\sim 0.2$ mag. A possible variation at $\sim 0.1 \mathrm{~d}$ superimposed on a decreasing longer-term trend might be also present (see e.g. the 2002 October 29 light curve). A combined linear plus sine fit to the October 29 light curve gives a period of $\sim 0.12 \mathrm{~d}$ and a semi-amplitude of $\sim 0.05 \mathrm{mag}$. To search for this and other periodicities in the light curves, we computed the Scargle periodogram after subtracting the nightly average values. The resulting power spectrum shows a group of strong peaks at low frequencies centred at approximately integer values and separated by $1 \mathrm{~d}^{-1}$ (see inset plot in Fig. 5). They are the result of the observing window. Two groups of peaks are located around $8 \mathrm{~d}^{-1}$ and $23 \mathrm{~d}^{-1}$. The centre of the strongest peak in each group was measured by fitting a Gaussian function to yield $0.127 \pm 0.002 \mathrm{~d}$ (in agreement with our initial estimate) and $0.0435 \pm 0.0002 \mathrm{~d}(62.7 \pm 0.3 \mathrm{~min})$.

We detrended the light curves of the longer term variation on which the $0.127-d$ wave lies. To do this, a smoothed version

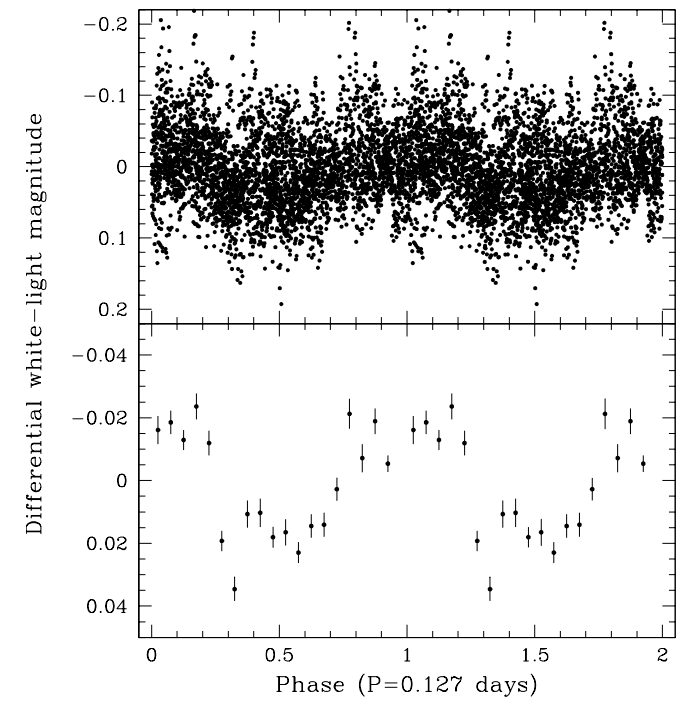

Fig. 6. CP Lac light curves folded on the 0.127 -d period after subtracting the long term trend (see text for details). The bottom panel shows the folded light curve after binning the data points into 20 phase intervals. A full cycle has been plotted twice for continuity.

of each light curve (using a 300-point boxcar) was subtracted to the original ones. We folded the detrended light curves on the $0.127-d$ period, obtaining the curve shown in Fig. 6 . The time of zero phase corresponds to the first acquired data point. The binned light curve shows a narrow dip at relative phase $\sim 0.3$ lasting for $\sim 25 \mathrm{~min}$. It would be desirable to have a longer photometric coverage to check whether this dip is an eclipse or not. Such a shallow eclipse (if present at all) can be lost in the similar amplitude, short-term variations observed in the light curve.

If the variation at $0.127 \mathrm{~d}$ is related to the orbital motion, CP Lac will lie just above the period gap, a region mainly inhabited by the SW Sex stars. The strength of the He II $\lambda 4686$ emission line and the rapid and non-coherent photometric variability in CP Lac also relate it to this class of CVs. Timeresolved spectroscopy of this fairly bright object will definitely shed more light on this issue.

\subsection{GI Mon}

GI Mon (Nova Monocerotis 1918) was a fast nova discovered on 1918 February 4 by M. Wolf after maximum brightness at $m_{\mathrm{pg}}=8.5$ (see e.g. Pickering 1918). The postnova has a magnitude of $V=16.2$ (Szkody 1994) and a blue spectrum with weak Balmer and He II $\lambda 4686$ emission lines (see Liu \& Hu 2000 and references therein). No nova shell was evident in the postnova spectrum at $\sim 6555 \AA$ (Gill \& O'Brien 1998).

We collected data on GI Mon during 2002 November 1 and $4(J K T)$ and 2004 March 17-18 (FLWO). The light curves are presented in Fig. 7. The 2002 November 4 light curve displays a $\sim 0.1$-mag, $\sim 45$-min long dip that could be interpreted as an eclipse. If this is the case, the absence of a similar dip in the other light curves would support a long orbital period. Additionally, the 2004 light curves show low amplitude, short 


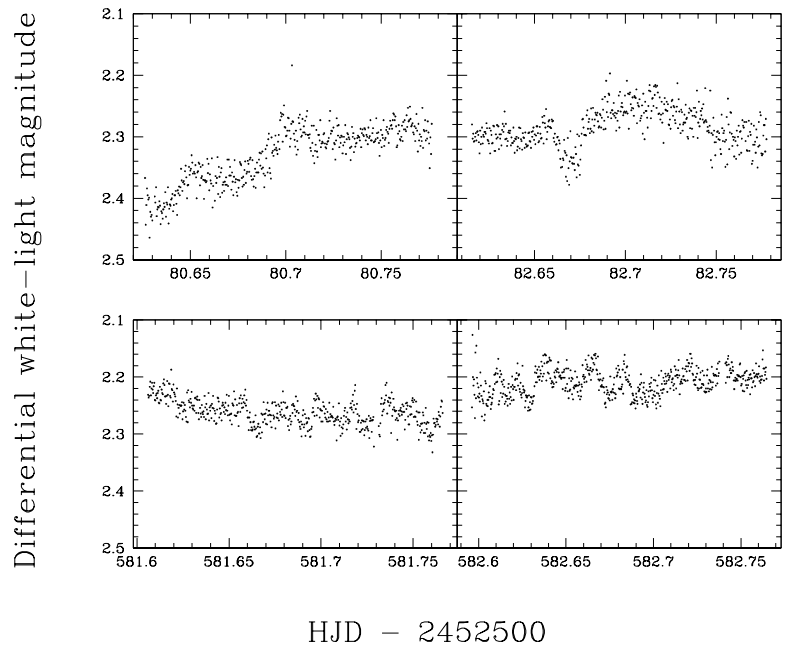

Fig. 7. Unfiltered light curves of GI mon obtained with the JKT (top panels; 2002 November 2, 4) and at the FLWO (bottom panels; 2004 March 17-18).

time scale $(\sim 10 \mathrm{~min})$ variations which are not present in the 2002 data.

One day after collecting our last data on GI Mon we were aware of the results of Woudt et al. (2004), who claim an orbital or superhump period of $0.1802 \mathrm{~d}$. Our periodogram from the 2002 dataset shows a cluster of peaks centred at approximately this period. However, there is no sign of it in the periodogram computed using our 2004 dataset despite the fact that the nightly observing windows are roughly the same in both years. Finally, the light curve labelled "S6155" in Fig. 9 of Woudt et al. (2004) shows a $~ 0.1$-mag dip with a duration of $\sim 40 \mathrm{~min}$, which is similar to the dip we found in our data.

\subsection{V400 Per}

V400 Per (Nova Persei 1974) was discovered spectroscopically after maximum at $m_{\mathrm{pg}} \sim 11$ on 1974 November 9 by Sanduleak (1974). Shao (1974) and Scovil (1974) independently identified the prenova with a faint blue star with $m_{\mathrm{pg}} \sim 19.5$. Examination of prediscovery photographic plates showed that the nova reached $V \sim 8$ on September 24 and there was no evidence for earlier outbursts during the period 1898.8-1952.8 (Shao 1974; Liller 1974). The nova light curve of V400 Per was in the limit of a moderately fast to slow nova (Mattei 1975; Landolt 1975). A $V$ magnitude of 19 for the postnova was reported in Szkody (1994).

It proved impossible to take useful images of V400 Per in white light and $2 \times 2$ binning due to the proximity (24.5" separation) of a bright star with $R \approx 11.0$ (USNO 1350 03079208). We then decided to use the Johnson $B$ filter to minimise its contribution, with the disadvantage of having to increase the exposure times. The CCD window was also chosen as to exclude as much of the contaminating star from the frames as possible.

The differential $B$-band light curves obtained on 2002 November 3 and $4(J K T)$, and on 2003 January 3 (IAC80) are presented in Fig. 8. The curve with longer coverage shows a modulation with a likely period of $\sim 0.16 \mathrm{~d}$ and

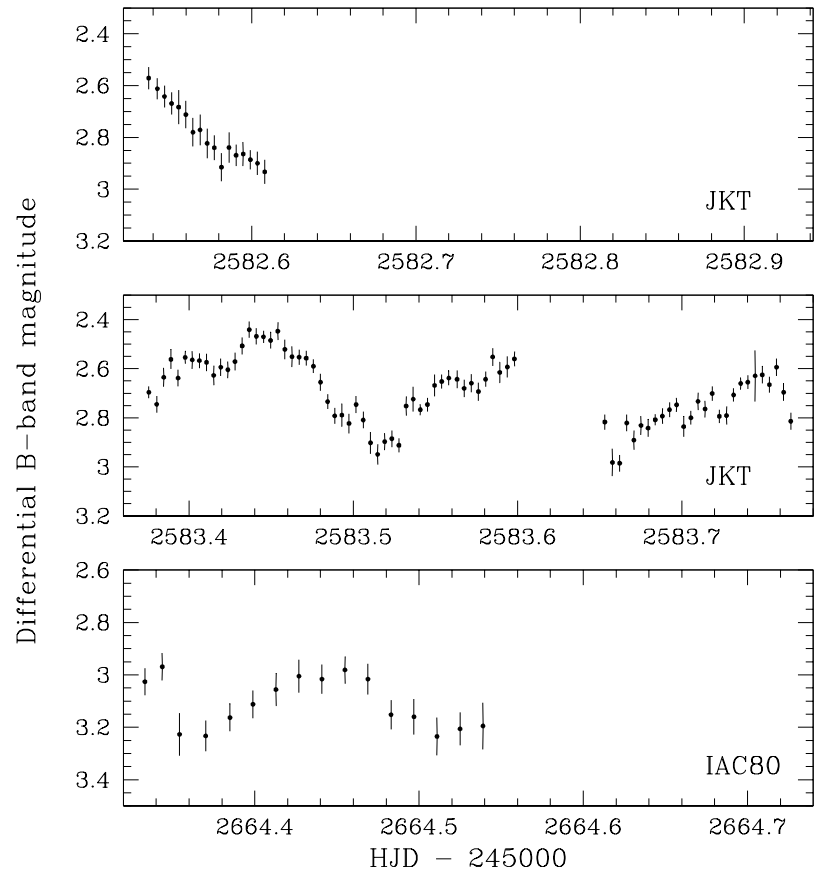

Fig. 8. $B$-band light curves of V400 Per. From top to bottom: 2002 November 3-4 (JKT), and 2003 January 3 (IAC80). A modulation with a period of $\sim 0.16 \mathrm{~d}$ can be seen in the curve with longest coverage.

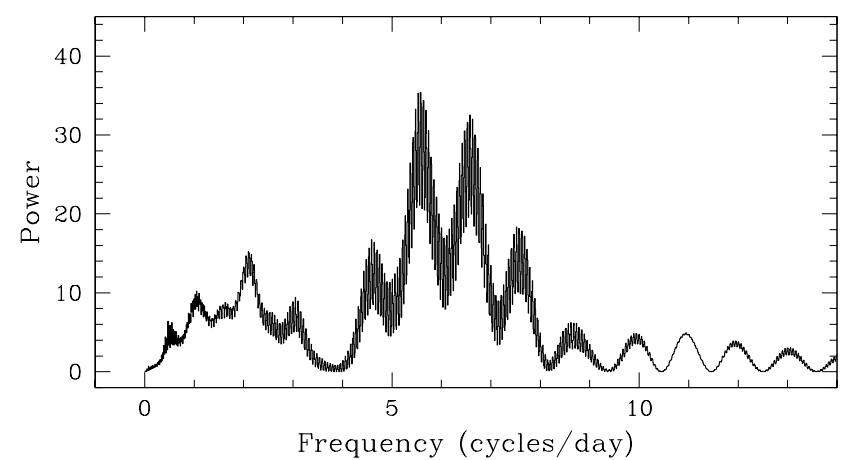

Fig. 9. Scargle periodogram computed from all the photometric data on V400 Per after subtracting the nightly averages.

a semi-amplitude of $\sim 0.2 \mathrm{mag}$. A sine fit to the IAC80 light curve provided a period of $0.16 \pm 0.02 \mathrm{~d}$ with a semi-amplitude of $0.12 \pm 0.02 \mathrm{mag}$. A Scargle periodogram computed from all the data (after subtracting the nightly means) is shown in Fig. 9. Two prominent peaks are seen in the frequency interval $5-7 \mathrm{~d}^{-1}$, centred at $5.58 \pm 0.03$ and $6.58 \pm 0.03 \mathrm{~d}^{-1}\left(\mathrm{a} 1 \mathrm{~d}^{-1}\right.$ alias). These frequencies correspond to the periods $0.179 \pm$ $0.001 \mathrm{~d}$ and $0.152 \pm 0.001 \mathrm{~d}$, respectively. Unfortunately, the amount of data available is insufficient to provide an unambiguous period. The observed modulation is likely related to the binary orbital motion, either as an orbital modulation or a superhump.

\subsection{CT Ser}

CT Ser (Nova Serpentis 1948) was likely a slow nova discovered after maximum brightness at magnitude close to 9.0 


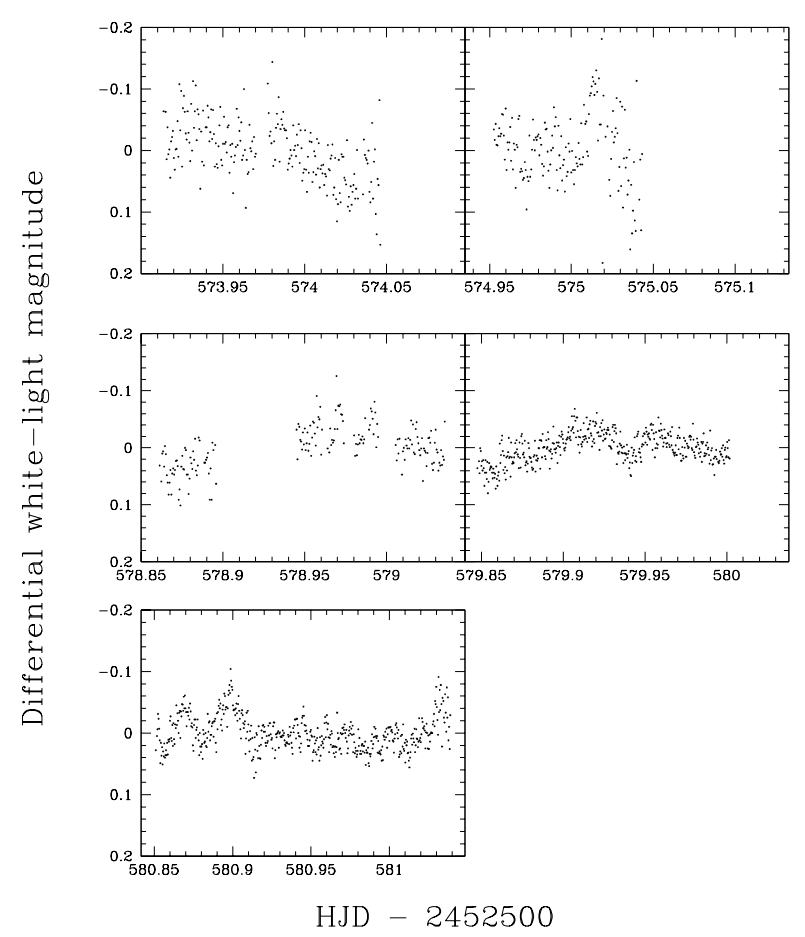

Fig. 10. Unfiltered light curves of CT Ser taken at the FLWO. From left to right and from top to bottom: 2004 March 9, 10, 14, 15 and 16.

by Bartay on 1948 April 9 (see McLaughlin 1948). From the analysis of a 2-h postnova light curve, Howell et al. (1990) reported photometric periodicites at 13 and $65 \mathrm{~min}$, suggesting an orbital origin to the latter. Additionally, Hoard et al. (2002) found a significative change in the near-IR colours of CT Ser with respect to those reported in Harrison (1992) from observations obtained six years earlier. This is explained as due to a transient phenomenon rather than to colour evolution. The postnova optical spectrum of CT Ser shows a blue continuum with Balmer and He II $\lambda 4686$ lines in emission (Ringwald et al. 1996; Szkody \& Howell 1992). However, not all of the $\mathrm{H} \alpha$ emission has its origin in the binary system as shown by Downes \& Duerbeck (2000) who resolved the nova shell at this wavelength.

The light curves of CT Ser obtained on 2004 March 9-10 and 14-16 at the FLWO are shown in Fig. 10. The differential photometry was made with respect to different comparison stars so we have subtracted the nightly averages. The Scargle periodogram obtained from our data is shown in Fig. 11. The strongest peak is centred at $0.162 \pm 0.001 \mathrm{~d}$. Alas, we can not address any conclusion since our longest light curve only spans $\sim 0.19 \mathrm{~d}$ and the data are not widely spread in time. CT Ser also shows shorter time scale variability, but no clear peak is observed in the periodogram. Thus, these variations are likely due to erratic flickering.

\subsection{Tau}

XX Tau (Nova Tauri 1927) was discovered by Schwassmann \& Wachmann (1928). The light curve of this fast nova attained photographic magnitude $m_{\mathrm{pg}}=6$ and displayed $t_{2}=$ 24 days, $t_{3}=43$ days (see e.g. Downes \& Duerbeck 2000).

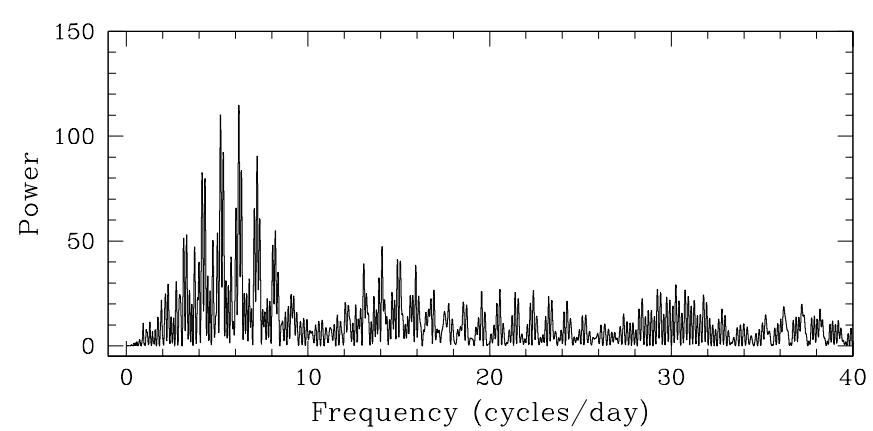

Fig. 11. Scargle periodogram for CT Ser after subtracting the nightly means.

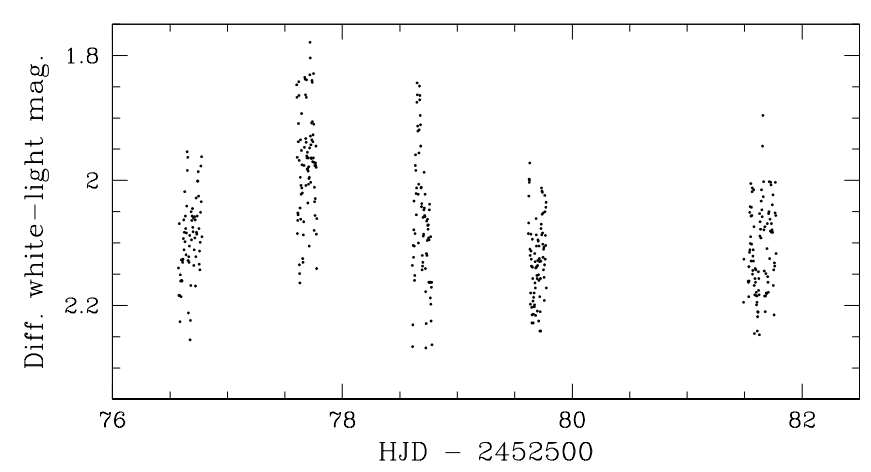

Fig. 12. Long-term light curve of $X X$ Tau in white light. The plot shows a clear variation of the mean brightness from night to night.

The postnova has an uncertain visual magnitude of $V=19.8$ (Szkody 1994).

The long term light curve of XX Tau is shown in Fig. 12. The mean brightness seems to be modulated with a periodicity of the order of a few days that could be associated to a long-term variation in the system such as that due to the precession of an eccentric/tilted accretion disc. No obvious periodic variability is observed in the individual light curves (Fig. 13). The observed scattering reflects the presence of a short time scale variation with an amplitude $\lesssim 0.2 \mathrm{mag}$. We have constructed a Scargle periodogram (Fig. 14) using all the available data. Most of the power is concentrated at about $7 \mathrm{~d}^{-1}$, with the strongest peak centred at $0.136 \pm 0.002 \mathrm{~d}$. The periodogram also shows significant power around $60 \mathrm{~d}^{-1}$, peaking at $0.01645 \pm 0.00002 \mathrm{~d}(23.69 \pm 0.03 \mathrm{~min})$. However, folding the data on $23.69 \mathrm{~min}$ (after removing the $0.136-\mathrm{d}$ modulation) does not yield a clear modulation and thereby we can be dealing with flickering or quasi-periodic oscillations rather than with a coherent signal.

The long term varibility of XX Tau may have a $\sim 5$-d periodicity. If this is the case, it will be similar to that observed in the SW Sex stars V442 Oph and RX J1643.7+3402 (Patterson et al. 2002), and PX And (Stanishev et al. 2002). These systems show a modulation of $4-5 \mathrm{~d}$ attributed to the precession of an eccentric/tilted disc and have orbital periods in the range $\sim 0.12-0.14 \mathrm{~d}$. Therefore, the modulation at $0.136 \mathrm{~d}$ we see in XX Tau could be related to the orbital period (e.g. a superhump period). 


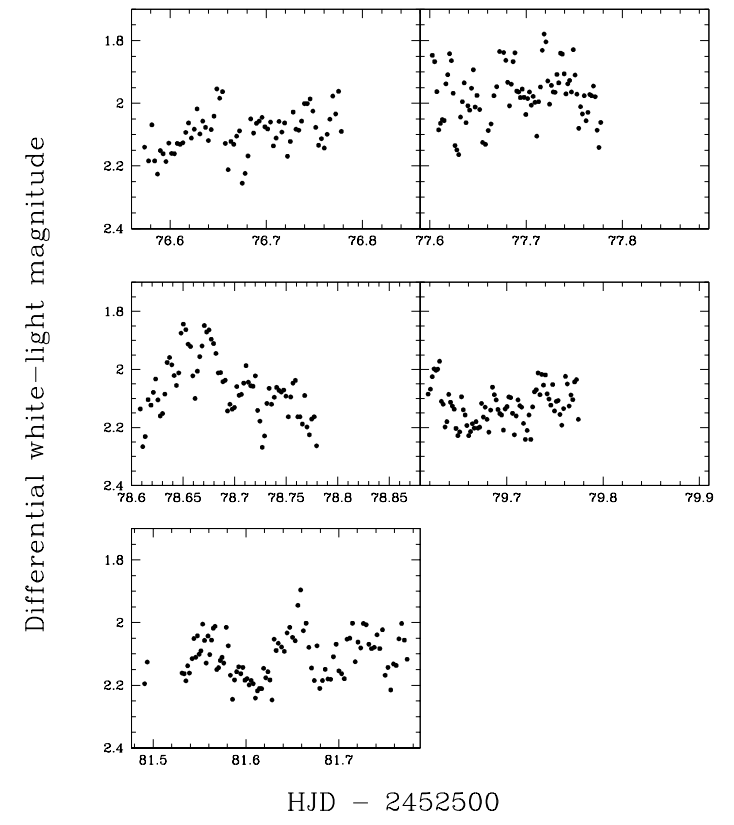

Fig. 13. Individual light curves of $\mathrm{XX}$ Tau obtained with the $J K T$. From left to right and from top to bottom: 2002 October 28-31 and November 2.

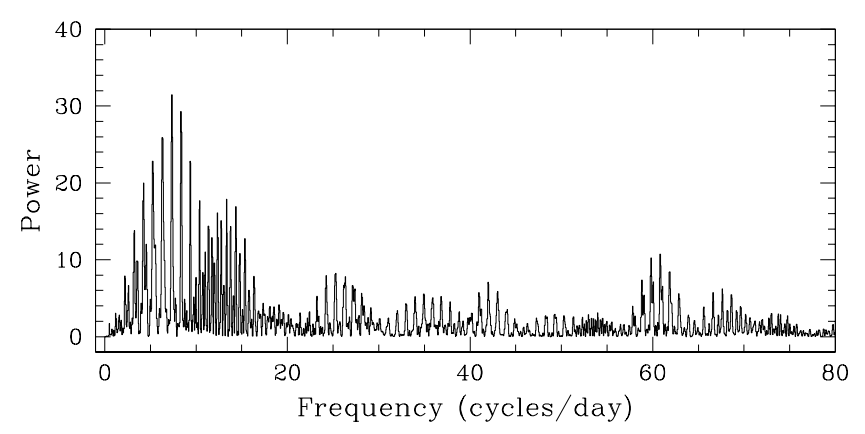

Fig. 14. Scargle periodogram for XX Tau.

\section{Conclusions}

In Fig. 15 we show all the periodograms presented in the paper (with the exception of GI Mon) so that the excess power for a given object can be easily spotted by comparison with the others. The likely periodicities found are summarised in Table 2.

\subsection{DM Gem}

The light curves provide a modulation at $0.123 \mathrm{~d}$ and possible rapid variability at $\sim 22 \mathrm{~min}$. The continuum slope of the optical spectrum suggests the presence of an accretion disc in the system. The strength of the He II $\lambda 4686$ emission line is comparable to that of $\mathrm{H} \beta$. The Bowen blend is also intense, indicating the presence of a source of ionising photons. We point out that DM Gem might be an IP or a SW Sex star. Future spectroscopic studies must have the appropriate time resolution to sample a possible $\sim 20$-min modulation in the emission-line flux and enough time coverage to properly deal with a $\sim 3-\mathrm{h}$ orbital period.

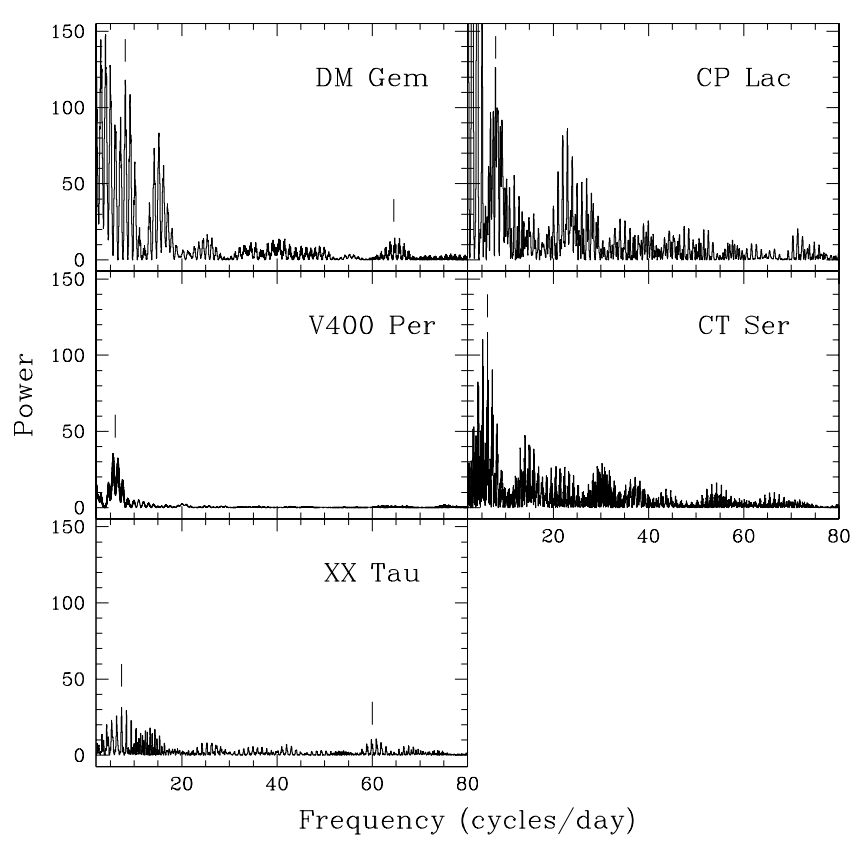

Fig. 15. All Scargle periodograms presented in the paper (with the exception of GI Mon) for easy comparison between objects. The peaks corresponding to the possible periodicities listed in Table 2 (excepting the long term variation in XX Tau) are marked with vertical lines.

\subsection{CP Lac}

This nova remnant is characterised by intense short time scale variability superimposed on a $0.127-\mathrm{d}$ wave. We tentatively suggest that shallow eclipses might be present. The intense He II $\lambda 4686$ and Bowen emission in its optical spectrum points towards a magnetic nature. If the $0.127-\mathrm{d}$ periodicity is related to the orbital motion, CP Lac will lie within the period range dominated by the SW Sex stars.

\subsection{GI Mon}

Our data are insufficient to confirm the 0.1802 -d periodicity reported by Woudt et al. (2004). We recorded a shallow dip in one of our light curves which must be confirmed as an eclipse. In the case GI Mon is an eclipsing CV, the length of our longest light curve constrains the orbital period to be $>0.2 \mathrm{~d}$.

\subsection{V400 Per}

Our $B$-band photometry provides a clear modulation at either 4.30 or $3.65 \mathrm{~h}$, which we link to the orbital motion of the binary.

\subsection{CT Ser}

The light curves do not provide a clear picture. A modulation at $\sim 0.17 \mathrm{~d}$ might be present. Erratic, short time scale variability is also observed. 
Table 2. Summary of possible periodicities.

\begin{tabular}{lll}
\hline \hline Object & Periodicity $(\mathrm{min})$ & Remarks \\
\hline DM Gem & $177.1,22.6$ & Strong He II 4 4686. Magnetic? \\
CP Lac & 182.9 & Shallow eclipses? SW Sex? \\
GI Mon & 259.2 & \\
V400 Per & 219 or 258 & \\
CT Ser & 233.3 & \\
XX Tau & $7200,195.8,23.7$ & Disc precession? \\
\hline
\end{tabular}

\subsection{Tau}

Our results favour a modulation at $\sim 0.14 \mathrm{~d}$ for this nova remnant. Fast variability at $\sim 24$ min might also be present, but it is uncertain. The most obvious feature is a brightness modulation at $\sim 5 \mathrm{~d}$, similar to other nova-like CVs (particularly the SW Sex stars). We attribute this long-period variation to the motion of an eccentric/tilted accretion disc in the binary.

Acknowledgements. We thank the anonymous referee for his/her valuable comments on the original manuscript. We are very grateful to Perry Berlind who kindly obtained the spectrum of DM Gem and to Emilio Falco for allocating two extra nights to our project. P.R.G. thanks PPARC for support through a PDRA grant. This work was supported in part by NASA grant NAG5-9930.

\section{References}

Bertaud, C. 1945, Ann. Paris Obs., 9, fasc. 1

Bode, M. F., \& Evans, A. 1989, Classical novae (Chichester: Wiley), ed. M. F. Bode, \& A. Evans

Cordova, F. A., Fenimore, E. E., Middleditch, J., \& Mason, K. O. 1983, ApJ, 265, 363

Downes, R. A., \& Duerbeck, H. W. 2000, AJ, 120, 2007

Fabricant, D., Cheimets, P., Caldwell, N., \& Geary, J. 1998, PASP, 110, 79

Gill, C. D., \& O’Brien, T. J. 1998, MNRAS, 300, 221

Harrison, T. E. 1992, MNRAS, 259, 17

Hoard, D. W., Wachter, S., Clark, L. L., \& Bowers, T. P. 2002, ApJ, 565,511
Honeycutt, R. K., Robertson, J. W., \& Turner, G. W. 1998, AJ, 115, 2527

Howell, S. B., Szkody, P., Kreidl, T. J., Mason, K. O., \& Puchnarewicz, E. M. 1990, PASP, 102, 758

Kurtz, M. J., \& Mink, D. J. 1998, PASP, 110, 934

Landolt, A. U. 1975, PASP, 87, 407

Liller, W. 1974, IAU Circ., 2729

Lipkin, Y., Leibowitz, E., \& Retter, A. 2000, IAU Circ., 7433

Liu, W., \& Hu, J. Y. 2000, ApJS, 128, 387

Mattei, J. A. 1975, JRASC, 69, 261

McLaughlin, D. B. 1948, PASP, 60, 265

Munari, U., \& Zwitter, T. 1997, A\&AS, 318, 269

Naylor, T. 1998, MNRAS, 296, 339

Patterson, J., Fenton, W. H., Thorstensen, J. R., et al. 2002, PASP, 114, 1364

Pickering, E. C. 1918, Harvard College Observatory Bulletin, 653, 1

Pickering, E. C., Fleming, W. P., \& Leland, E. F. 1903, ApJ, 17, 305

Ringwald, F. A., Naylor, T., \& Mukai, K. 1996, MNRAS, 281, 192

Robinson, E. L. 1975, AJ, 80, 515

Rodríguez-Gil, P. 2003, Ph.D. Thesis

Rodríguez-Gil, P., Casares, J., Martínez-Pais, I. G., Hakala, P., \& Steeghs, D. 2001a, ApJ, 548, L49

Rodríguez-Gil, P., Martínez-Pais, I. G., Casares, J., Villada, M., \& van Zyl, L. 2001b, MNRAS, 328, 903

Sanduleak, M. 1974, IAU Circ., 2716

Scargle, J. D. 1982, ApJ, 263, 835

Schmidt, T. 1957, Z. Astrophys., 41, 182

Schwassmann, A., \& Wachmann, A. 1928, Astron. Nachrichten, 232, 273

Scovil, C. 1974, IAU Circ., 2722

Shao, C. Y. 1974, IAU Circ., 2722

Stanishev, V., Kraicheva, Z., Boffin, H. M. J., \& Genkov, V. 2002, A\&A, 394, 625

Starrfield, S., Sparks, W. M., \& Truran, J. W. 1976, in Structure and Evolution of Close Binary Systems, IAU Symp., 73, 155

Steiner, J. E., Schwartz, D. A., Jablonski, F. J., et al. 1981, ApJ, 249, L21

Szkody, P. 1994, AJ, 108, 639

Szkody, P., \& Howell, S. B. 1992, ApJS, 78, 537

Warner, B. 1995, Cataclysmic Variable Stars (Cambridge: Cambridge University Press)

Williams, G. 1983, ApJS, 53, 523

Woudt, P. A., Warner, B., \& Pretorius, M. 2004, MNRAS, 351, 1015 